\title{
Access to subsidized artemether-lumefantrine from the private sector among febrile children in rural setting in Kilosa, Tanzania
}

\section{DAUDI SIMBA ${ }^{1}$ and DEODATUS KAKOKO ${ }^{2}$}

${ }^{1}$ Department of Community Health, Muhimbili University of Health and Allied Sciences, P. O. Box 65015, Dar es Salaam, Tanzania

2Department of Behavioural Sciences, Muhimbili University of Health and Allied Sciences, Dar es Salaam, Tanzania

\begin{abstract}
The World Health Organization aims at universal access to effective antimalarial treatment by the year 2015. Consequently, an enormous financial resource has been invested on Artemisinin Combination Therapy (ACT))) subsidy. In Tanzania, strategies to increase access of artemether-lumufantrine (ALu) rural areas, where the burden is highest, includes subsidy to the Faith-based Organisations (FBO) facilities and accredited drug dispensing outlets (ADDOs). This study was done to assess the extent to which children suffering from malaria access ALu from the private sector in rural areas. A total of 1,235 under fives randomly selected from 12 rural villages were followed up at home on weekly basis for six months in Kilosa district in 2008. Using a structured questionnaire, caretakers were interviewed about the child's history of fever in the past 7 days; type of treatment given and the source. Baseline data were obtained on demographic characteristics, caretakers' knowledge about malaria and social economic indicators of the household. Of the 1,235 children followed-up, 740 care-seeking visits were recorded, of which, $264(35.7 \%)$ were made at government health facilities and nearly a quarter $(24.1 \% ; 178 / 740)$ at ordinary shops that sell general merchandize including rice and sugar. Only $22 \%$ of the caretakers sought care from FBO and ADDOs. While $686(86.6 \%)$ of the episodes were treated with antimalarials, only $319(43 \%)$ received ALu, the recommended antimalarial. Majority $(83 \%)$ of the visits made at government facilities were prescribed with ALu compared to less than half who went to FBO facilities (40.0\%) and ADDOs (25.0\%). In conclusion, this study has shown that less than a quarter of fever episodes suspected to be malaria in rural areas were made at FBO facilities and ADDOs, of which, less than half were treated with ALu. This shows that ALu subsidy to formal private sector does not adequately reach children in rural areas, where the malaria burden is highest. This cast some doubts if the target of universal access to effective antimalarial, by 2015, will be reached. There is need to consider enlisting the services of community health workers in the efforts to improve access to ALu in rural areas. Further research is needed to explore providers' / dispensers' preference for non-recommended antimalarials in the private sector and caretakers' preference for ordinary shops.
\end{abstract}

Keywords: malaria, Artemisinin-combination therapy, health services, accessibility, Tanzania

\section{Introduction}

Nearly half of the world's population lives in geographic areas where malaria is present. In 2009, about 243 million cases of malaria occurred worldwide claiming about 863,000 lives (WHO, 2009). About $90 \%$ of these deaths occurred in Africa, and about $80 \%$ were among children under the age of five. The main strategy for the reduction of these deaths is prompt treatment using effective drugs, currently, Artemisinin-based combined therapy. The WHO target to achieve universal access to effective antimalarials by the year 2015 (WHO, 2009). An enormous amount of fund has been raised through the Global Fund for AIDS/HIV, Tuberculosis and Malaria (GF-ATM) in the effort to achieve this goal, between 2003 and 2006 the amount of money earmarked for malaria interventions increased by six-fold from 3 billion to 17 billion (WHO, 2009). However, these efforts are constrained by the

\footnotetext{
${ }^{1}$ Correspondence: Daudi Simba; E-mail: daudisimba@yahoo.com; dsimba@muhas.ac.tz
} 
inadequate health systems in many malaria endemic countries (Task force on health systems research, 2004). The health systems in these countries are characterised by poor infrastructure, human resources as well as logistics and information systems. The rural remote areas in these countries are affected most, partly because of the higher level of poverty (National Bureau of Statistics, 2002a) and longer distance to health facilities compared to urban areas (Simba et al., 2010).

The change of malaria treatment policy from sulphadoxine-pyrimethamine (SP) to artemetherlumefantrine $(\mathrm{ALu})$ in Tanzania saw the restriction in dispensing $\mathrm{ALu}$ in order to avoid misuse that might lead to drug resistance (Price et al., 2006). At the time of this study, ALu was provided free of charge in government facilities and subsidized price at the faith-based organization (FBO) facilities and drug shops accredited to sell a selected number of prescription only drugs, the facilities are known as ADDOs (Moon et al., 2009). Since 2010, ALu has been available at a subsidized price through all registered dispensing outlets through subsidy at manufacturer's level (Alba et al., 2010, Arnold et al., 2012).

Despite the subsidized price strategy, access to ALu by children with fever suspected to be due to malaria has been quite low. A survey conducted in 12 regions of Tanzania reported only $22 \%$ of the children accessed ALu (MoHSW, 2008a). A study done in Kenya, soon after the implementation of the drug policy change, reported only $10 \%$ of the febrile children accessed ACT, promptly (Gitonga et al., 2008). The majority of children who had an access to ACT were the ones who sought care from government health facilities, where services are provided free of charge for children. However, more than half of the febrile children were not taken to government health facilities (Simba et al., 2010). Several studies have reported caretakers' preference for private facilities over government facilities when seeking for care (Munguti, 1998; Noor et al., 2003; Rutebemberwa et al., 2009b) because of convenience. Government health facilities have been reported to have long waiting times, unfriendly provider-patient interactions and constant drug shortages.

This paper report findings from a study that was conducted to determine the extent to which children suffering from malaria access subsidized ALu from the private sector in rural areas.

\section{Materials and Methods}

\section{Study area}

The study was conducted in Kilosa district in Tanzania between June and November 2008. Malaria transmission in the district occurs throughout the year. The district has a population of about 488,000 people (National Bureau of Statistics, 2002b). There are 257 villages in the district of which 135 are in rural areas. There were 71 health facilities in the district of which 61 were dispensaries, 7 health centres and 3 hospitals (Kilosa District Council, 2008). Of these, Faith-based organisations (FBO) own two hospitals, one health centres and nine dispensaries. There were 152 drug shops that were licensed to sell non-prescription drugs; although this regulation was not always enforced. Six months prior to this study, a new initiative was introduced in which about 92 drug shops in the district were transformed into Accredited Drug Dispensing Outlets (ADDOs). Drug dispensers from these facilities were trained on principles of drug dispensing before they were allowed to sell a selected number of prescription-only drugs including ALu (Price et al. 2006), the only ACT available in the district (MoHSW, 2008b). Whereas, government facilities provided the drug free of charge, FBO facilities and ADDOs sold at a subsidised price. Drug shops and ordinary shops that sell general merchandize such as rice and sugar also served as sources of antimalarials in the district, but did not stock ALu. 


\section{Study design, sample size and selection}

A total of 1,235 children under the age of five were followed up weekly for six months to establish the source of treatment and the type of drugs, for those reported to have had fever suspected to be due to malaria. At that time, the malaria treatment guidelines in the country recommended treating febrile children with effective antimalarial, that was, ALu using presumptive diagnosis of malaria (MoHSW, 2006).

The sample size was calculated using 95\% confidence interval $+/-5 \%$ units, assuming a proportion of $50 \%$. A $10 \%$ addition was made for possible loss to follow-up and $38 \%$ as correcting factor for clustering effect on village level (ICC=0.01), The sample size came to 583; and using a 2week incidence rate of $6 \%$ reported in Kilombero, Tanzania (Hetzel et al., 2008), we needed to followup 1,200 children for 16 weeks. Twelve villages were selected out of the 135 rural villages using stratified random sampling whereby; three villages were selected from each of the four geographical areas in the district which were wet-highland, dry-highland, wet-lowland and dry-lowland. From each area, one village with a health facility and two villages without were selected. Children were selected from the villages according to village population size. Village registers were updated and used to select children, randomly, using Epi-Info, software. In the course of the study, 35 children were replaced because they had either shifted to other villages or completed the age of five, making the number of children followed up 1,235.

\section{Data collection procedure}

Three research assistants were recruited to collect baseline data that included demographic and socioeconomic data. Village health workers serving in the respective villages were recruited and trained for one week on administration of a simple questionnaire. They followed up enrolled children at home every Saturdays and Sundays to identify those who developed fever in the past 7 days to establish the source of care, treatment received and the cost incurred, if any. Medical notes and drug packets were used to ascertain the type of drug provided. Written informed consents were obtained from the caretakers prior to interviews.

\section{Data analysis}

Data entry was done using EpiData 3.0 (EpiData Association. Odense, Denmark) and analysed using STATA version 10.1 (Stata Corp., College station, TX, USA). Data validation was done through double-entry and inconsistency check using frequency and two by two tables. Analysis was performed on the type of drug received by source of care. The median expenditure on drugs was analysed by source of care.

\section{Ethical considerations}

Ethical clearance was obtained from the Institutional Review Board of the Muhimbili University of Health and Allied Sciences and permission to conduct the study was obtained from the regional, district and village authorities.

\section{Results}

A total of 1,235 children were followed up for a six-month period, of which, a total of 970 episodes of fever were recorded; 740 were first episodes. Since we were not able to establish whether $2^{\text {nd }}$ and $3^{\text {rd }}$ episodes were new infection or recurrent we based the analysis on first episodes only. Table 1 shows 
that, of the 740 care-seeking visits, $264(35.7 \%)$ were made at government health facilities. Nearly a quarter $(24.1 \%$; $178 / 740)$ of the visits was made at ordinary shops. Less than a quarter of the visits were made at FBO facilities (11.6\%) and ADDOs (10.0\%).

Table 1: Source of care for children suffering from fever suspected to be malaria

\begin{tabular}{lll}
\hline Source of care & Number of care-seeking visits made & Percent \\
\hline Government & 264 & 35.6 \\
Ordinary Shops* $^{*}$ & 178 & 24.1 \\
Home care with modern drugs & 89 & 12.0 \\
Faith-Based Organization facilities & 86 & 11.6 \\
Drug shops & 36 & 4.9 \\
Traditional healers/medicines & 33 & 4.5 \\
Accredited Drug Dispensing Outlets & 28 & 3.8 \\
General home care¥ & 24 & 3.2 \\
Private health facilities & 2 & 0.3 \\
Total & $\mathbf{7 4 0}$ & $\mathbf{1 0 0 . 0}$ \\
\hline
\end{tabular}

Key: *-selling general merchandize such as sugar and rice; ¥-included tepid sponging and bathing

Of the 740 care-seeking visits, antimalarials were prescribed in 686 (86.6\%) of the episodes (Table 2). However, ALu was prescribed in only 319 (43\%) of all the visits (Table 2). Whereas, in the majority of the visits made at government health facilities children were prescribed ALu, in less than half of the visits made at FBO facilities (40.0\%) and ADDOs (25.0\%), respectively, children received ALu. Altogether, FBO facilities and ADDOs prescribed / dispensed about 36\% (41/114) of the ALu received by children.

Table 2: Type of drug prescribed or dispensed to febrile children

\begin{tabular}{|c|c|c|c|c|c|c|c|c|c|c|c|c|c|c|}
\hline \multirow{2}{*}{$\begin{array}{l}\text { Type of drug } \\
\text { Prescribed/ } \\
\text { dispensed }\end{array}$} & \multicolumn{2}{|c|}{ Government } & \multicolumn{2}{|c|}{ FBO } & \multicolumn{2}{|c|}{ ADDO } & \multicolumn{2}{|c|}{ Others } & \multicolumn{2}{|c|}{ Shops } & \multicolumn{2}{|c|}{ Home care } & \multicolumn{2}{|c|}{ Total } \\
\hline & $\mathbf{n}$ & $\%$ & $\mathbf{n}$ & $\%$ & $\mathbf{n}$ & $\%$ & $\mathbf{n}$ & $\%$ & $\mathbf{n}$ & $\%$ & $\mathbf{n}$ & $\%$ & $\mathbf{n}$ & $\%$ \\
\hline $\mathrm{ALu}$ & 220 & 83 & 34 & 40 & 7 & 25 & 5 & 12 & 13 & 7.3 & 40 & 28 & 319 & 43 \\
\hline SP & 11 & 4.2 & 9 & 10 & 8 & 29 & 12 & 28 & 79 & 44 & 35 & 25 & 154 & 21 \\
\hline Amodiaquine & 5 & 1.9 & 14 & 16 & 2 & 7.1 & 17 & 40 & 48 & 27 & 20 & 14 & 106 & 14 \\
\hline Quinine & 15 & 5.7 & 18 & 21 & 7 & 25 & 6 & 14 & 5 & 2.8 & 13 & 9.2 & 64 & 8.6 \\
\hline Antipyretic & 3 & 1.1 & 1 & 1.2 & 1 & 3.6 & 2 & 4.7 & 20 & 11 & 17 & 12 & 44 & 5.9 \\
\hline Don't know & 6 & 2.3 & 7 & 8.1 & 2 & 7.1 & 0 & 0 & 1 & 0.6 & 10 & 7.1 & 26 & 3.5 \\
\hline Others & 4 & 1.5 & 3 & 3.5 & 1 & 3.6 & 1 & 2.3 & 12 & 6.7 & 6 & 4.3 & 27 & 3.6 \\
\hline Total & 264 & 100 & 86 & 100 & 28 & 100 & 43 & 100 & 178 & 100 & 141 & 100 & 740 & 100 \\
\hline
\end{tabular}

The second commonest dispensed antimalarial was Sulphadoxine-Pyrimethamine (SP) $(21.0 \%$; 154/740) followed by amodiaquine (14.0\%; 106/740). SP accounted for about one third $(36.6 \% ; 154 / 421)$ 
of the non-ALu prescriptions while Amodiaquine accounted for a quarter $(25.2 \%$; 106/421) of the prescription. SP or amodiaquine was dispensed in the majority of the episodes $(71.1 \% ; 127 / 178)$ at the ordinary shops, accounting for nearly half $(48.9 \% ; 127 / 260)$ of the non-recommended antimalarials prescribed / dispensed. Incidentally, some of the ALu were dispensed at ordinary shops (7.3\%; 13/178).

Caretakers reported paying for drugs in 628 of the episodes of which 34 of them were not able to report the cost because payments were handled by someone else. The median expenditure on drugs was highest in FBO facilities (median TSh 2500; range TSh 0-10,000) and lowest in government facilities (median 0; range 0-3,100) (Table 3). The expenditure on drugs at ADDOs was higher (median TSh 1,600) compared to drug shops (median TSh 900).

Table 3: Costs (in Tanzania shillings) incurred by caretakers at various drug outlets

\begin{tabular}{llllll}
\hline Source of drugs & $\begin{array}{l}\text { Number of } \\
\text { caretakers }\end{array}$ & Median & Mean & Minimum & Maximum \\
\hline Faith-based organization facilities & 86 & 2500 & 2815 & 0 & 10000 \\
Private facilities & 2 & 500 & 2500 & 500 & 4500 \\
Accredited Drug Dispensing Outlets & 28 & 1600 & 1701 & 200 & 5000 \\
Drug shops & 36 & 900 & 1115 & 50 & 3000 \\
Ordinary shops & 178 & 500 & 878 & 0 & 8500 \\
Government facilities & 264 & 0 & 78 & 0 & 3100 \\
Total/Average & $\mathbf{5 9 4}$ & $\mathbf{2 0 0}$ & $\mathbf{8 0 5}$ & $\mathbf{0}$ & $\mathbf{1 0 0 0 0}$ \\
\hline
\end{tabular}

Key: US dollar 1 = Tanzanian Shillings 1,200

\section{Discussion}

This study has shown that FBO facilities and ADDOs prescribed / dispensed ALu in less than half of the episodes of fever suspected to be due to malaria. A majority of the febrile children received SP or amodiaquine drugs that were not recommended for treatment of uncomplicated malaria (WHO, 2009). This finding confirms previous studies where most of the patients attending private sector facilities were reported to have received ineffective antimalarials (Chuma et al., 2009; Goodman et al., 2007; Rutebemberwa et al., 2009a). The findings from this study is an indication that the strategy to reduce the price of $\mathrm{ALu}$ through subsidy might not necessarily ensure access to ALu to febrile children residing in rural areas, where the burden of malaria is the highest and the level of poverty is equally high.

In relation to the findings of this study, it is plausible to ask the question; why caretakers seeking care at FBO facilities and ADDOs where ALu is available at subsidized price were less likely to get the drug? Accordingly, several reasons can be brought up to explain this finding. A study done in Zambia reported health workers' reluctance to prescribe ALu was due to concerns about drug potency, side effects, or concerns about interruptions to the stock (Zurovac et al., 2005). The availability of SP and amodiaquine in the market might also serve as an inducement for health workers to prescribe the drug. Dispensers in drug shops are often guided by caretaker's request for specific drugs without necessarily having to produce a prescription (Goodman et al., 2007). On the other hand, caregivers' might have preferred alternative antimalarials due to the trust they have on 
the drugs and the relative low price compared to subsidized ALu. Drug shops are reported to honour patients' choice when dispensing medicines (Viberg, 2009).

This study has shown that majority of febrile children were taken to the private sector where the majority preferred ordinary shops rather than FBO facilities and ADDOs. The low attendance at FBO facilities is likely to be explained by the small number of FBO facilities in the district. In addition, caretakers might have utilized FBO facilities less frequently because of the relatively high cost charged in these facilities. The high cost has reportedly been attributed to the need to recover operating costs (Njau et al., 2006). Low attendance to ADDOs might be explained by the location of these facilities. ADDOs and drugs shops are normally located in semi-urban and urban areas, where the high population density ensures a better income compared to rural areas. In addition, studies have reported that people normally utilise facilities that are closer before opting for those that are far away (Munguti, 1998; Noor et al., 2003). Since ordinary shops are relatively closer to people where they don't incur transport costs and they sell drugs at cheaper prices, caretakers' preference for ordinary shops is therefore not surprising. In addition to proximity and low prices, caretakers might have preferred ordinary shops as a source of 'first aid'. Caretakers have been reported to perceive malaria as a mild condition and to resort to seeking care from ordinary shops and only go to other facilities when the condition is perceived to be serious (Comoro et al., 2003; de Savigny et al., 2004; Warsame et al., 2007).

Findings from this study can be generalised to other rural districts in the country and elsewhere that shares similar characteristics to Kilosa district with respect to difficulty in reaching health facilities due to rough roads, wide geographical areas, inadequate availability of drug shops with large populations residing more than $5 \mathrm{~km}$ from a nearby public health facility.

Findings from this study should be interpreted with caution because not all episodes of fever were due to malaria. The use of presumptive malaria diagnosis in this study might result into overdiagnosis of malaria. However, at the time of this study, the national malaria treatment guidelines recommended treatment of malaria based on presumptive diagnosis of malaria. Further research is needed now that rapid diagnostic tests have been introduced countrywide.

In conclusion, this study has shown that less than a quarter fever episodes suspected to be due to malaria in rural areas were made at FBO facilities and ADDOs, of which, less than half were treated with ALu. This shows that ALu subsidy to formal private sector does not adequately reach children in rural areas, where the malaria burden is highest. This cast some doubts if the target of universal access to effective antimalarial, by 2015, will be reached. Further research is needed to establish the reasons for providers' / dispensers' preference for non-recommended antimalarials in FBO facilities and ADDOs and caretakers' preference for ordinary shops. Meanwhile, there is need to consider enlisting the services of community health workers, residing in the rural areas, in the efforts to improve access to ALu in rural areas, especially, those remote from health facilities.

\section{Acknowledgments}

We are grateful to the Muhimbili University of Health and Allied Sciences (MUHAS)/ Karolinska Institute (KI) collaboration for the support in conducting the study that led to this paper and Sida/SAREC for funding. Thanks to Amos Massele for ideas and administrative support in the designing and implementation of the study. Finally, we wish to thank all participants, research assistants and others people who made the study possible. 


\section{References}

Alba, S., Hetzel, M., Goodman, C., Dillip, A., Liana, J., Mshinda, H. \& Lengeler, C. (2010) Improvements in access to malaria treatment in Tanzania after switch to artemisinin combination therapy and the introduction of accredited drug dispensing outlets - a provider perspective. Malaria Journal, 9, doi:10.1186/1475-2875-9-163

Arnold, F., Ye, Y., Ren, R., Hanson, K., Goodman, C., Tougher, S., A., M. \& Willey, B. (2012) Independent Evaluation of Phase 1 of the Affordable Medicines Facility - malaria (AMFm): Multi-Country Baseline Report submitted to Global Fund.

Chuma, J., Abuya, T., Memusi, D., Juma, E., Akhwale, W., Ntwiga, J., Nyandigisi, A., Tetteh, G., Shretta, R. \& Amin, A. (2009) Reviewing the literature on access to prompt and effective malaria treatment in Kenya: implications for meeting the Abuja targets. Malaria Journal 8, doi:10.1186/1475-2875-8-243.

Comoro, C., Nsimba, S. E., Warsame, M. \& Tomson, G. (2003) Local understanding, perceptions and reported practices of mothers/guardians and health workers on childhood malaria in a Tanzanian district--implications for malaria control. Acta Tropica 87, 305-13.

De Savigny D, Mayombana C, Mwageni E, Masanja H, Minhaj A, Mkilindi Y, Mbuya C, Kasale H \& G., R. (2004) Care-seeking patterns for fatal malaria in Tanzania. Malaria Journal 3:27.

Gitonga, C., Amin, A., Ajanga, A., Kangwana, B., Noor, A. \& R, S. (2008) The use of artemetherlumefantrine by febrile children following national implementation of a revised drug policy in Kenya. Tropical Medicine and International Health 13, 487-494.

Goodman, C., Kachur, P., Abdulla, S., Bloland, P. \& Mills, A. (2007) Drug shop regulation and malaria treatment in Tanzania-why do shops break the rules, and does it matter? Health Policy and Planning, 1, doi:10.1093/heapol/czm1033.

Hetzel, M., Alba, S., Fankhauser, M., Mayumana, I., Lengeler, C., Obrist, B., Nathan, R., Makemba, A., Mshana, C., Schulze, A. \& Mshinda, H. (2008) Malaria risk and access to prevention and treatment in the paddies of the Kilombero Valley, Tanzania. Malaria Journal 7, doi:10.1186/1475-2875-7-7

Kilosa District Council (2008) Council Annual Report - 2008 (Unpublished).

MoHSW (2006) National Guidelines for Malaria Diagnosis and Treatment 2006. Ministry of Health and Social Welfare, Dar es Salaam, Tanzania.

MoHSW (2008a) National Malaria Control Programme - Medium Term Malaria Strategic Plan 2008 - 2013. Ministry of Health and Social Welfare, Dar es Salaam, Tanzania.

MoHSW (2008b) Stakeholders' Meeting Report on Accredited Drug Dispensing Outlets Program. Ministry of Health and Social Welfare, Dar es Salaam, Tanzania.

Moon, S., Casas, C., Kindermans, J., De Smet, M. \& Von Schoen-Angerer, T. (2009) Focusing on Quality Patient Care in the New Global Subsidy for Malaria Medicines. PLoS Medicine 6, e1000106.

Munguti, K. (1998) Community perceptions and treatment seeking for malaria in Baringo district, Kenya: implications for disease control. East African Medical Journal 75, 687-691.

National Bureau of Statistics (2002a) Household budget survey. (Unpublished)

National Bureau of Statistics (2002b) Tanzania Census - Analytical report Volume X. Dar es Salaam, Ministry of Planning, Economy and Empowerment.

Njau, J., Goodman, C., Kachur, S., Palmer, N., Khatib, R., Abdulla, S., Mills, A. \& Bloland, P. (2006) Fever treatment and household wealth: the challenge posed for rolling out combination 
therapy for malaria. Tropical Medicine and International Health 11, doi:10.1111/j.13653156..01569.x.

Noor, A., Zurovac, D., Hay, S., Ochola, S. \& Snow, R. (2003) Defining equity in physical access to clinical services using geographical information systems as part of malaria planning and monitoring in Kenya. Tropical Medicine and International Health 8, 917-926.

Price, R., Uhlemann, A., Van, V., Brockman, A., Hutagalung, R., Nair, S., Nash, D., Singhasivanon, P., Anderson, T., Krishna, S., White, N. \& Nosten, F. (2006) Molecular and pharmacological determinants of the therapeutic response to artemether-lumefantrine in multidrug-resistant Plasmodium falciparum malaria. Clinical Infectious Disease 42, 1570-1577.

Rutebemberwa, E., Kallander, K., Tomson, G., Peterson, S. \& Pariyo, G. (2009a) Determinants of delay in care-seeking for febrile children in eastern Uganda. Tropical Medicine and International Health $14,472-479$.

Rutebemberwa, E., Pariyo, G., Peterson, S., Tomson, G. \& Kallander, K. (2009b) Utilization of public or private health care providers by febrile children after user fee removal in Uganda. Malaria Journal 8, doi:10.1186/1475-2875-8-45.

Simba, D., Warsame, M., Kakoko, D., Mrango, Z., Tomson, G., Premji, Z. \& Petzold, M. (2010) Who Gets Prompt Access to Artemisinin-Based Combination Therapy? A Prospective CommunityBased Study in Children from Rural Kilosa, Tanzania. PLoS ONE 5, e12104. doi:10.1371/journal.pone.0012104.

Task Force on Health Systems Research (2004) Informed choice for attaining the Millennium Development Goals: towards an international cooperative agenda for health systems research. Lancet 364, 997-1003.

Viberg, N., Mujinja, P., Kalala, W., Kumaranayake, L., Vyas, S., Tomson, G. And Lundborg, C. (2009) STI management in Tanzanian private drugstores -practices and roles of drugsellers. Sexually Transmitted Infections doi:10.1136/sti.2008.032888.

Warsame, M., Kimbute, O., Machinda, Z., Ruddy, P., Melkisedick, M., Peto, T., Ribeiro, I., Kitua, A., Tomson, G. \& Gomes, M. (2007) Recognition, perceptions and treatment practices for severe malaria in rural Tanzania: implications for accessing rectal artesunate as a pre-referral. PLoS ONE 2, e149.

WHO (2009) World Malaria Report. World Health Organization, Geneva.

Zurovac, D., Ndhlovu, M., Rowe, A., Hamer, D., Thea, D. \& Snow, R. (2005) Treatment of paediatric malaria during a period of drug transition to artemether-lumefantrine in Zambia: cross sectional study. British Medical Journal 331, 734 